\title{
MPPT Control Strategy of PV Based on Improved Shuffled Frog Leaping Algorithm under Complex Environments
}

\author{
Xiaohua Nie ${ }^{1}$ and Haoyao Nie $^{2}$ \\ ${ }^{1}$ Information Engineering School, Nanchang University, Nanchang, Jiangxi Province 330031, China \\ ${ }^{2}$ Economics Management School, Nanchang University, Nanchang, Jiangxi Province 330031, China \\ Correspondence should be addressed to Xiaohua Nie; niexiaoh@163.com
}

Received 19 January 2017; Revised 22 May 2017; Accepted 14 June 2017; Published 15 August 2017

Academic Editor: Daniela Proto

Copyright (c) 2017 Xiaohua Nie and Haoyao Nie. This is an open access article distributed under the Creative Commons Attribution License, which permits unrestricted use, distribution, and reproduction in any medium, provided the original work is properly cited.

\begin{abstract}
This work presents a maximum power point tracking (MPPT) based on the particle swarm optimization (PSO) improved shuffled frog leaping algorithm (PSFLA). The swarm intelligence algorithm (SIA) has vast computing ability. The MPPT control strategies of PV array based on SIA are attracting considerable interests. Firstly, the PSFLA was proposed by adding the inertia weight factor $w$ of PSO in standard SFLA to overcome the defect of falling into the partial optimal solutions and slow convergence speed. The proposed PSFLA algorithm increased calculation speed and excellent global search capability of MPPT. Then, the PSFLA was applied to MPPT to solve the multiple extreme point problems of nonlinear optimization. Secondly, for the problems of MPPT under complex environments, a new MPPT strategy of the PSFLA combined with recursive least square filtering was proposed to overcome the measurement noise effects on MPPT accuracy. Finally, the simulation comparisons between PSFLA and SFLA algorithm were developed. The experiment and comparison between PSLFA and PSO algorithm under complex environment were executed. The simulation and experiment results indicate that the proposed MPPT control strategy based on PSFLA can suppress the measurement noise effects effectively and improve the PV array efficiency.
\end{abstract}

\section{Introduction}

The MPPT technology is one of the key PV technologies in the application of PV. To further improve the accuracy of MPPT is the eternal pursuit of MPPT, whose aim is to maximize the conversion of solar energy into electric energy. Due to the PV array itself, the DC/DC converter, and other complex environment factors, the nonlinear $V$ - $P$ curve of PV not only is characterized by multiple maximum points but also causes the problem of measurement noise and outliers, which reduce the accuracy of MPPT and the efficiency of PV $[1,2]$.

With the development of intelligent and optimization algorithms, they have been widely used in various fields of industrial electrical appliances [2, 3]. In recent years, many GMPPT (Global Maximum Power Point Tracking) algorithms have been proposed to deal with the failure of conventional MPPT algorithm because of the existence of multiple power maxima. These GMPPT algorithms improved the tracking accuracy effectively $[4,5]$, but they were effective without considering the measurement noise and outliers; it has been proved by simulation analysis that the measurement noise and outliers have influence on MPPT accuracy [6]. The performance of the MPPT algorithm is limited by measurement noises and outliers, and many GMPPT algorithms would be useless in practical application because the measurement noise and outliers are very important factors to be considered for the optimization of MPPT. Based on the above analysis, the validity of the existing GMPPT algorithms still has to be verified with measurement noises and outliers. Therefore, the MPPT strategy based on filtering is proposed, such as Kalman filter [7] or least square filter [8]. These MPPT strategies with filters can inhibit or reduce the influence of measurement noises and improve the tracking accuracy and response speed.

With vast computing ability, the MPPT based on swarm intelligence algorithm (SIA) are attracting considerable interests. The SIA is a kind of bionic search algorithm that includes particle swarm optimization (PSO), Shuffled Frog Leap Algorithm (SFLA), ant colony optimization (ACO), and artificial bee colony $(\mathrm{ABC})$ algorithm. The main advantages 
of SIA are (1) adapting to the environment automatically, (2) parallel searching, (3) global optimization, and (4) strong robustness, (5) being easy to combine with other algorithms. There are many papers introducing the application of the PSO, ACO, and ABC to MPPT [9-13], which reflected the effectiveness and feasibility of MPPT strategy based on SIA. Despite the advantages, these MPPT strategies also cause some defects: if the $V$-P curves create abnormal measurement outliers, these algorithms based on standard SIA without filtering algorithms would be useless. To overcome this problem, this paper preprocesses abnormal measurement outliers through the recursive least square filtering and then executes the MPPT control by the PSO improved shuffled frog leaping algorithm (PSFLA).

The SFLA algorithm is a heuristic evolutionary algorithm by imitating foraging behavior of frogs, proposed by Dr. Eusuff in 2003; it has been applied to solve water pipe optimization problem successfully [14]. Compared with PSO, ACO, GA, and MA [15], SFLA is similar to PSO in the aspect of global optimization but has the advantages of less parameters and fast calculation. It has succeeded in applying to the combinatorial optimization [16], reactive power optimization of power system [17], parameter identification [18], and many other fields since the algorithm was proposed which reflects its powerful search ability and robustness. However, there are not any study reports on the SFLA algorithm applied to PV MPPT yet.

The volt-power $(V-P)$ curve of PV array is not only characterized by multiple maxima points but also characterized by the measurement noise caused by the restriction of actual application under the complex environments, which was ignored by most of the recent literature on MPPT control strategy. In this paper, first, the PSFLA was proposed by adding the inertia weight factor $w$ of PSO in standard SFLA to overcome the defect of falling into the partial optimal solutions and slow convergence speed of SFLA. Then, the PSFLA was applied to MPPT to solve the multi-extreme point problems of nonlinear optimization. Second, for the problems of MPPT under complex environments, a new MPPT strategy of the PSFLA combined with recursive least square filtering was proposed to reduce the measurement noise influence. Finally, the simulation comparison between PSFLA and SFLA was developed. The experiment and comparison between PSLFA and PSO algorithm under complex environment were executed.

\section{The Improved Shuffled Frog Leaping Algorithm with PSO}

2.1. Standard Shuffled Frog Leaping Algorithm. The SFLA is a new metaheuristic population evolutionary algorithm and it has fast calculation speed and excellent global search capability. It has the advantages of simple concept, few parameters, fast calculation speed, strong global optimization ability, and so on.

SFLA solve problem with the collaboration and communication behavior shown in the foraging process. In SFLA, each frog is defined as a solution to the problem and the whole frog population is divided into many different small groups called meme group to simulate the clustering behavior; each frog group has the ability of judging food source which is affected by other groups. In each evolutionary process, the best and the worst frog in each group were located and the worst frog was updated by some way which is similar to the velocity shift model operator in PSO. After a certain number of evolutions in each group, all the frog groups are mixed into the whole frog population to exchange and share the information among each updated frog group. These steps will perform again and again until a predetermined number of times have been executed.

The algorithm flow of standard SFLA is as follows: first, the SFLA parameters are initialized, and a frog population $P$ representing the solution of the problem is generated randomly and sorted in a descending order according to fitness value of each frog. Then, the entire population is divided into $m$ meme group, each containing $n$ frogs (i.e., $P=m \times n)$. In this process, the first frog goes to the first meme group, the second frog goes to the second meme group, frog $m$ goes to the $m$ th meme group, frog $m+1$ goes back to the first meme group, and so on. Within each meme group, the frogs with the best and the worst fitness are identified as $F_{b}$ and $F_{w}$, respectively. Also, the frog with the global best fitness is identified as $F_{g}$. Then, a process similar to PSO is applied to improve the frog with the worst fitness (not all frogs) in each cycle. Accordingly, the position of the frog with the worst fitness is adjusted as follows:

Change in frog position is

$$
D=r *\left(F_{b}-F_{w}\right)
$$

New position is

$$
F_{w}^{\text {new }}=F_{w}+D, \quad\|D\| \leq D_{\max }
$$

where $r$ is a random number between 0 and 1 and $D_{\max }$ is the maximum allowed change in frog position. If this process produces a better solution, $F_{w}^{\text {new }}$ replaces the worst frog. Otherwise, repeat the calculations in (1) and (2) replacing the global best frog (i.e., $F_{g}$ replaces $F_{b}$ ). If no improvement becomes possible in this case, then a new solution is randomly generated to replace $F_{w}$. The calculations then continue for a specific number of iterations. The main feature of SFLA is that the whole population is divided into many different meme groups. The SFLA execute the local search and update the worst frog only; on the contrary, the PSO execute the global search and update each individual. So, the SFLA reduce the computation effectively and improve the efficiency compared with the PSO.

\subsection{Improve the Standard Shuffled Frog Leaping Algorithm} with PSO. The standard SFLA leapfrog rule which is just updating the worst frog can be described as shown in Figure 1(a). This unique rule improves the execution speed effectively, but $F_{w}^{\text {new }}$ is restricted to the position between $F_{w}$ and $F_{b}[19] ; F_{w}^{\text {new }}$ is more likely to be in the vicinity of $F_{b}$ (the shadow part of Figure $1(\mathrm{~b})$ ) in fact; in the meantime, $F_{w}$ 's historical optimal value $\left(F_{h}\right)$ is helpful to the updating of $F_{w}$ [20].

In addition, although SFLA is updating frog by meme group dividing, the optimization process in each meme 


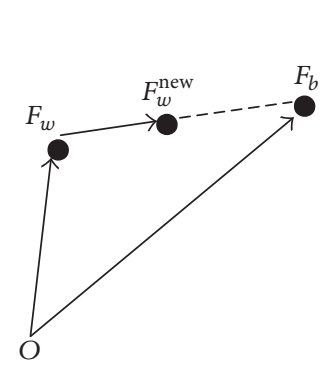

(a)

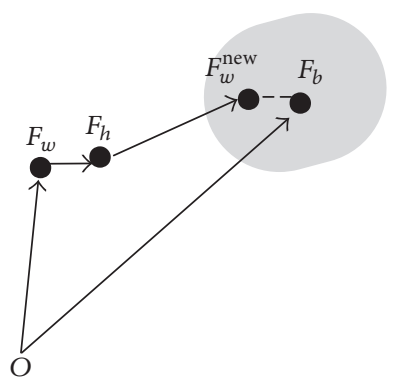

(b)
FIgURE 1: The rules schematic of leapfrog.

group is still a global optimization process just like PSO [21]. Therefore, we hope that the algorithm has good global search performance at the beginning and can locate the region near the global optimum quickly, and the latter part of the algorithm has good local search performance and can locate the global optimal solution accurately. $w$ is the most important parameter of PSO algorithm, which has great influences on the performance and efficiency of the algorithm: $w$ is the inertia weight and determined the global and local search capability of the PSO algorithm. A larger $w$ benefits PSO global search, and a small $w$ benefits local search. Considering the effect of the inertia weight factor $w$ to the performance of PSO, it is introduced in standard SFLA to improve the global search performance. The adjusting rule is described as follows:

Change in frog position is

$$
\begin{aligned}
& w_{i}=w_{\max }-\left(\frac{\left(w_{\max }-w_{\min }\right)}{G}\right) * i, \\
& D=w_{i} *\left(F_{b}-F_{w}\right) .
\end{aligned}
$$

New position is

$$
F_{w}^{\text {new }}=\left(F_{h}+r *\left(F_{b}-F_{h}\right)\right) \pm D \quad D_{\max } \leq D \leq D_{\text {min }},
$$

where $r$ is a random number between 0 and $1, G$ is the iteration numbers of local search in each meme group, and $i$ is the current iteration number. The PSFLA take the random point between $F_{h}$ and $F_{b}$ as starting point; take the difference between $F_{b}$ and $F_{w}$ as the step size. Experience shows that a linear gradient of inertia weight from 0.9 to 0.1 can gain better algorithm performance [22], setting $w_{\max }=0.9$ and $w_{\min }=0.1$.

\section{The MPPT Control Strategy Based on PSFLA}

The output characteristic of PV under complex environments has the problems of (1) multiple maxima points, which would bring about the failure of conventional MPPT algorithm because of tracking error and fall into the partial power maxima when standard SIA was adopted, (2) measurement noises and outliers, which reduced the accuracy of MPPT and the efficiency of PV, and (3) repeat voltage points. For example, in the case of repeat voltage points, the MPPT control methods based on SIA will fail because they cannot determine which voltage value is the right. The questions mentioned happened in our experiment and had not been mentioned in other research papers of MPPT based on SIA yet.

These characteristics have a significant impact on the performance of MPPT strategies based on the standard SIA without filtering algorithms optimization. To solve the existing problems, the solutions we can take are as follows. (1) It is necessary to suppress the noise and the outliers before MPPT and the recursive least square filtering method is used to data preprocessing in this paper. (2) Taking the duty cycle as control variable (horizontal coordinates), the maximum power point is not affected by taking the duty cycle as control variable.

To make up the deficiency of the existing MPPT algorithm, recursive least square filtering was used to weaken or inhibit the measurement noise and outliers, and the PSFLA was applied to MPPT control strategy. The tracking flow of the proposed MPPT control strategy based on PSFLA combined with recursive least square filtering was shown in Figure 2.

3.1. Data Filtering with Recursive Least Square Algorithm. The MPPT process of PV is modeled as a dynamic system by using the state-space method and is described as follows:

$$
\begin{aligned}
& P(k)=P(k-1)+w(k-1), \\
& Z(k)=P(k)+v(k),
\end{aligned}
$$

where $w(k-1)$ and $v(k-1)$ are represented by the state error and measurement error and the variances are $Q$ and $R$, respectively. In the actual data acquisition, the power value is calculated by the measured voltage and current rather than being measured directly. That is,

$$
\begin{aligned}
Z(k) & =\left[U(k)+\sigma_{u}\right]\left[I(k)+\sigma_{i}\right] \\
& \approx P(k)+U(k) \sigma_{i}+I(k) \sigma_{u},
\end{aligned}
$$

where $\sigma_{u}$ and $\sigma_{i}$ are measurement errors of the voltage and current, respectively.

In this paper, the sensor measurement error of the voltage and current both are $1 \%$ and the range of sensor measurement is $[0,100 \mathrm{~V}]$ and $[0,2 \mathrm{~A}]$, respectively. The maximum errors of $\sigma_{u}$ and $\sigma_{i}$ are $1.0 \mathrm{~V}$ and $0.02 \mathrm{~A}$, and the maximum measurement error of the power $\sigma_{P}$ is

$$
\begin{aligned}
\sigma_{P}^{2} & =\left[\sigma_{i} \times \max \left(U_{k}\right)+\sigma_{u} \times \max \left(I_{k}\right)\right]^{2} \\
& =[0.02 \times 100+1.0 \times 2]^{2}=4^{2} .
\end{aligned}
$$

In order to include the extreme case in the control process, the maximum value of $\sigma_{P}^{2}$ is $4^{2}$.

The least square estimation of $P(t)$ in $k$ th moment is determined by

$$
\widehat{P}(k)=\frac{1}{k} \sum_{t=1}^{k} P(t) .
$$




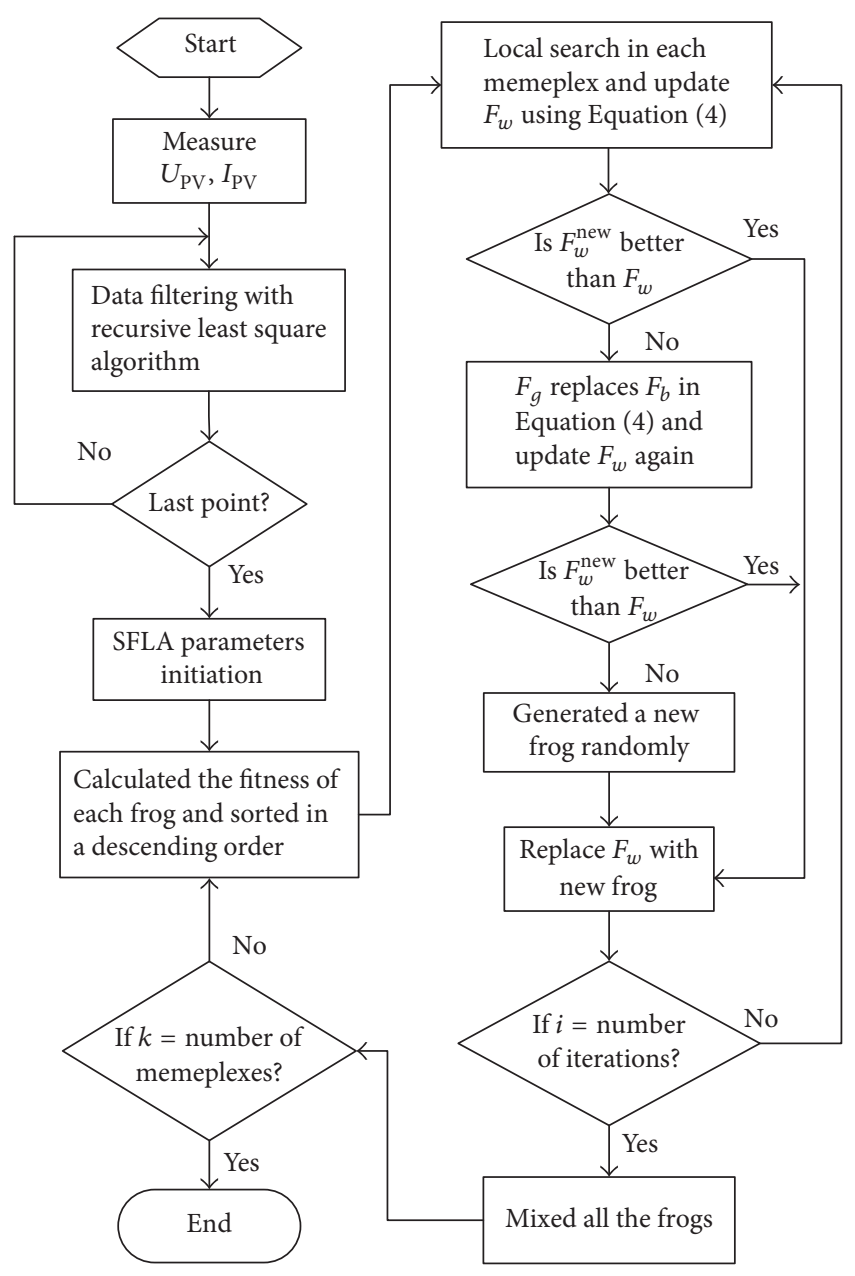

FIgURE 2: The flowchart of MPPT.

The estimation variance is

$$
\widehat{\sigma}_{P}^{2}=\frac{\sum_{t-1}^{k}[P(k)-\widehat{P}(k)]^{2}}{k} .
$$

Then get $P(t+1)$ and the recursive value of $P(t+1)$ at $(k+1)$ th moment is

$$
\widehat{P}(k+1)=\widehat{P}(k)+\frac{1}{k+1}[Z(k+1)-\widehat{P}(k)] .
$$

The instantaneous power is estimated by the above recursive least square filtering algorithm. In order to prevent the outliers caused by measurement error and tracking the power mutation caused by the abrupt change of the light correctly, it needs to eliminate the outliers and state judging.

Assume that

$$
d(k)=P(t+1)-\widehat{P}(k+1) .
$$

Set the threshold value of outlier $\left(\delta_{k}\right)$ as

$$
\delta_{k}=3 \widehat{\sigma}_{P}
$$

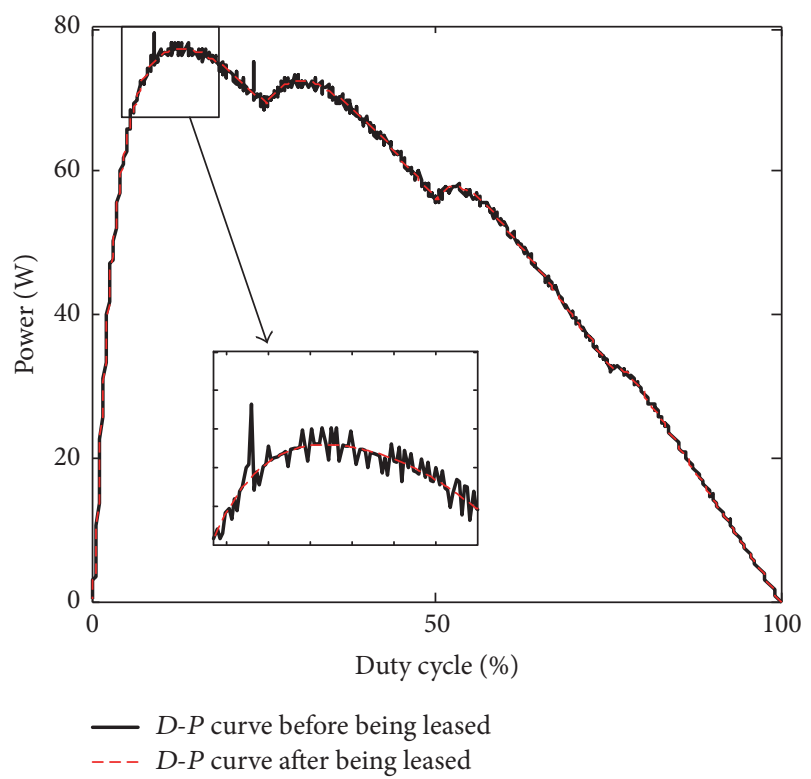

Figure 3: $D-P$ characteristics after least square of PV.

Power can be considered change if $|d(k)|$ is bigger than the threshold value.

The duty cycle-power $(D-P)$ characteristic curve of PV array is shown in Figure 3; the measurement noise and outliers are suppressed effectively.

\subsection{The Performance of PSLFA in MPPT Control Strategy. In} the MPPT strategy based on PSFLA, the fitness value was defined as output power of PV array and the frog position represents the duty cycle $D$. The parameter setting based on the $D-P$ characteristic curve of PV can make the MPPT more time-efficient and the tracking process more intuitive.

(1) Parameter Setting. The main parameters of PSFLA include number of frogs $N$; number of meme groups $m$; number of generations for each meme group before shuffling $M$; number of shuffling iterations MaxIter; and step size $D$. The value of these parameters is related to the search performance of PSFLA: the bigger the value of $N$, the bigger the possibility of PSFLA locating the global optimum point, but as $N=$ $m * n$, the values of $m, n$ are coupled together with $N$ which affects the search efficiency seriously; as for $M$, the small value will make the remix after a few times of evolution which will weaken the local search ability; on the contrary, it will increase the search time and lead to premature convergence easily. There is no relevant theoretical basis on how to set the parameters. By comparing several simulation results, the parameters were set as follows: $N=200, m=20, M=10$, and MaxIter $=100$ and the range of $D$ is set to $[0,1]$.

(2) Termination Strategy. In order to reduce the power loss caused by multiple iterations after the algorithm located the global maximum power point, it is essential to take the corresponding termination strategy. Considering that $F_{g}$ (i.e., the frog with the global best fitness) remains unchanged 

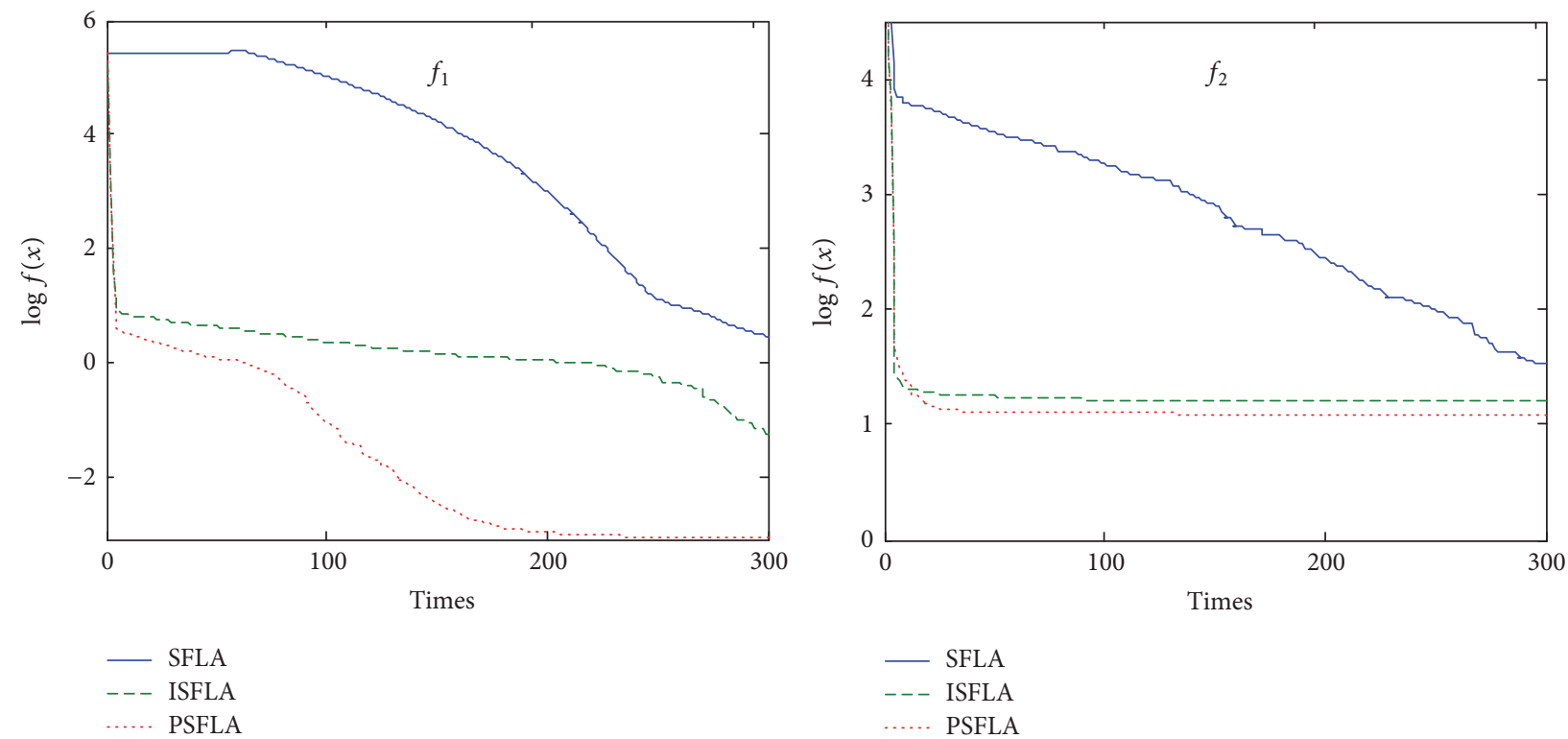

FIGURE 4: The evolutionary curves.

in subsequent iterations after locating the global maximum power point, the termination strategy is set as follows: we consider that the global maximum power point has been located if $F_{g}$ remains unchanged in the continuous 20 iterations, and then stop iteration.

\section{Simulation and Experiment}

4.1. Simulation and Comparison between PSLFA, ISFLA, and SFLA Algorithm. In order to verify the effectiveness of the proposed MPPT strategy based on PSFLA combined with recursive least square filtering whose goal is to overcome the effect of the complex environments, three simulation experiments and one small power prototype experiment were designed. Simulation 1: two commonly testing functions were selected to compare the performance of the standard SFLA, the improved SFLA (ISFLA) in [18] and the PSFLA without filtering algorithms in this paper; Simulation 2: the PSFLA is used in MPPT just in the case of partial shading condition (PSC); Simulation 3: we combine PSFLA with recursive least square filtering and used it in MPPT in the case of complex environments.

4.1.1. Simulation and Comparison 1. The testing function is

$$
\begin{aligned}
& f_{1}(x)=1+\sum_{i=1}^{N} \frac{x_{i}^{2}}{4000}-\prod_{i=1}^{N}\left(\cos \left(\frac{x_{i}}{\sqrt{i}}\right)\right) \\
& \quad\left(-600 \leq x_{i} \leq 600\right), \\
& f_{2}(x)=\sum_{i=1}^{N}\left(x_{i}^{2}+x_{i+1}^{2}\right)^{0.25} \\
& \cdot\left(\left(\sin \left(50 *\left(x_{i}^{2}+x_{i+1}^{2}\right)^{0.1}\right)\right)^{2}+1\right) \\
& \quad\left(-100 \leq x_{i} \leq 100\right) .
\end{aligned}
$$

$f_{1}$ is Griewank's function and $f_{2}$ is Schaffer function; the two functions have a large number of local extreme points which makes the traditional optimization algorithm difficult to search the global optimal solution. The global optimum solution (minimum) for these two functions is known to be zero when all variables equal zero, the values of $f_{1}$ 's variable are constrained to a range ( -600 to 600$)$, and $f_{2}$ 's are ranging from -100 to 100 .

The parameters of SFLA are set as follows [18]: $N$ is 300 and $m$ is $30, M$ is 20 and MaxIter is 300, the dimension of the solution is 30 , and each algorithm runs 200 times independently. The experiment result is shown in Table 1, and the evolutionary curves are shown in Figure 4.

We can see that the PSFLA have better performance in both the accuracy and speed than the SFLA and ISFLA from the test results of Table 1 and Figure 4.

4.1.2. Simulation and Comparison 2. During partial shading condition, the D-P curves are characterized by multiple peaks (several local and one global peak (GP)). At this point, the PSFLA without filtering algorithms was used in MPPT and the power of the optimal frog in the iterative process changes as shown in Figure 5. The global optimal frog is no longer changed after a certain number of evolutions and the maximum power is $77.25 \mathrm{~W}$. Compared with the actual maximum power which is $77.23 \mathrm{~W}$, the difference is only $0.02 \mathrm{~W}$ which indicates that the PSFLA without filtering algorithms can locate the GP from multiple peaks under partial shading condition.

4.1.3. Simulation and Comparison 3. Under complex environments, the $D-P$ curves are characterized by not only multiple peaks, but also measurement noise and outliers caused by practical application limits. In this case, the PSFLA without filtering algorithms and the PSFLA combined with recursive 
TABLE 1: The experimental results.

\begin{tabular}{lcccc}
\hline Function & Algorithm & Minimum & $\begin{array}{c}\text { Mean } \\
\text { value }\end{array}$ & $\begin{array}{c}\text { Standard } \\
\text { deviation }\end{array}$ \\
\hline \multirow{2}{*}{$f_{1}$} & SFLA & 1.1915 & 2.0379 & 0.4842 \\
& ISFLA & 0.0050 & 0.1793 & 0.1562 \\
& PSFLA & $8.84 E-06$ & 0.0495 & 0.137 \\
\hline \multirow{2}{*}{$f_{2}$} & SFLA & 20.763 & 61.5609 & 8.6011 \\
& ISFLA & 6.5754 & 15.3768 & 1.2074 \\
& PSFLA & 5.8960 & 18.2074 & 1.3768 \\
\hline
\end{tabular}

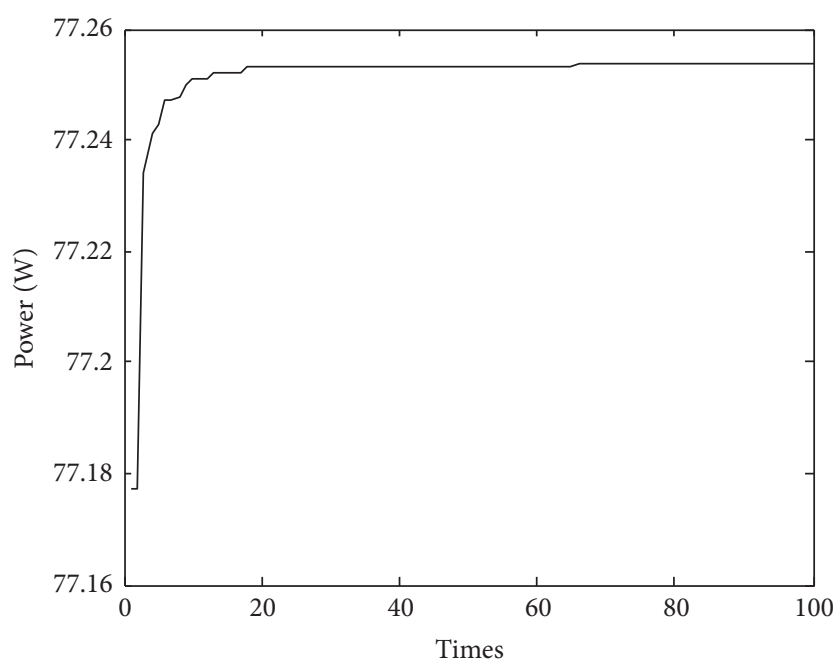

FIGURE 5: The power of PV array during search.

least square filtering were used in MPPT, respectively, the parameters of PSFLA were described in Section 3.2, each algorithm runs 200 times independently, and the results are shown in Figure 6.

Without combining the recursive least square filtering, the power located by the PSFLA is ranging from $77.89 \mathrm{~W}$ to $80.89 \mathrm{~W}$ under the influence of measurement noise and outliers, the mean value is $79.55 \mathrm{~W}$, and the relative error is $3 \%$ which means that MPPT failed because of the effect of measurement noise and outliers. On the contrary, combining the PSFLA with recursive least square filtering, the power is ranging from $77.59 \mathrm{~W}$ to $76.98 \mathrm{~W}$ which is not much different from the theoretical value. The PSFLA combined with the recursive least square filtering improves the accuracy of MPPT overcoming the effect of the complex environments effectively. Based on the above analysis, we can get that the proposed MPPT strategy based on PSFLA combined with recursive least square filtering is effective; it can locate the global maximum power under the complex environments and improve the search accuracy of MPPT.

\subsection{Experiment and Comparison}

4.2.1. Experimental Platform. In order to verify the practicability of the proposed MPPT strategy in this paper, a MPPT experimental platform based on K60 (the chip model

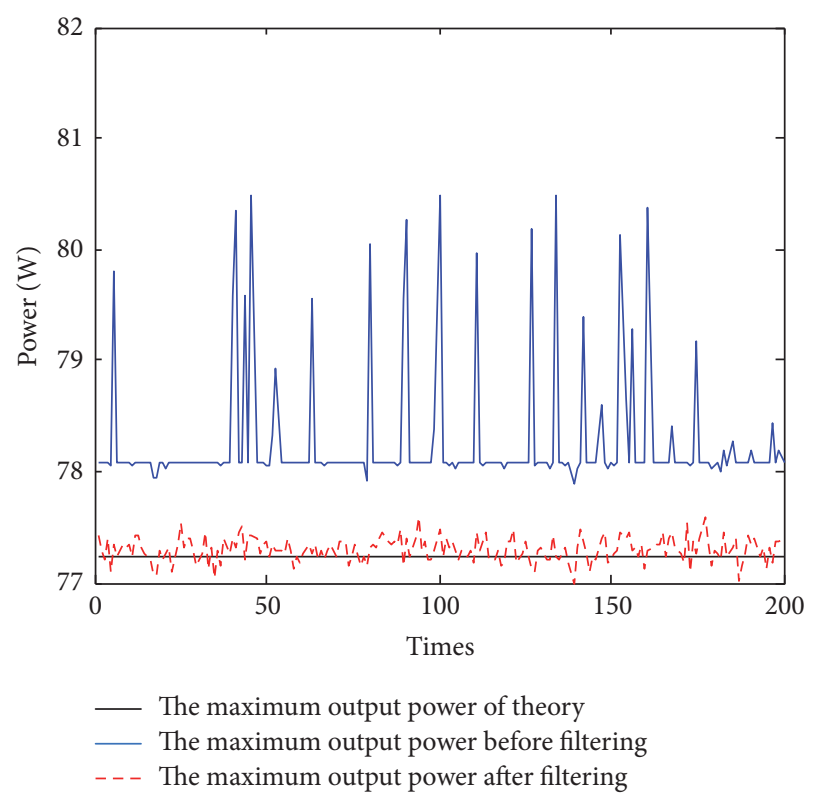

FIGURE 6: Comparison of tracking results.

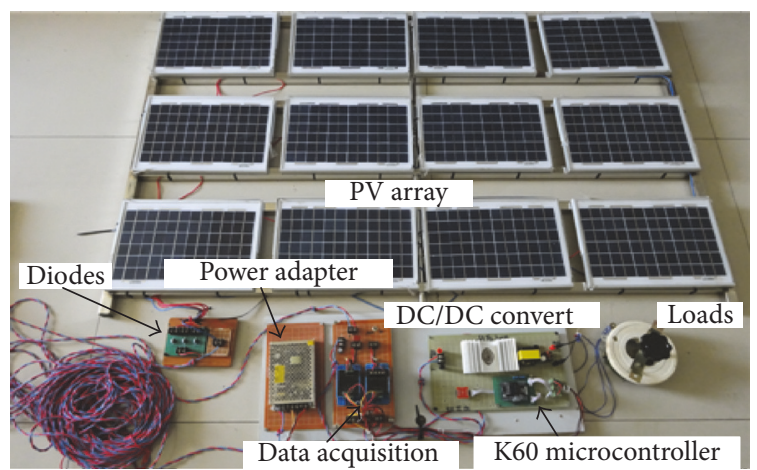

FiguRE 7: Experimental platform.

is K60P100SYS) microcontroller was established as shown in Figure 7 and the MPPT control principle was shown in Figure 8 . The PV array is set to $3 \times 4$ connection structure as shown in Figure 9 and its detailed parameters of solar cells are shown in Table 2.

The circuit consists of PV array, boost circuit, and MPPT controller. The parameters of the boost circuit are set as follows: $L=4 \mathrm{mH}, C_{2}=470 \mathrm{uF}$, and $f 2=30 \mathrm{kHz}$; the load is a resistor. The voltage and current are real time sampled by Hall sensor and then are sent to the K60 microcontroller where the data will be calculated to get the right duty cycle. The main circuit is controlled by the duty cycle so it can regulate the output power of PV array. In this paper, the voltage-power curves of PV array are sampled and scanned every 5 minutes. Then, the maximum power point is calculated and optimized by the swarm intelligence algorithms. The experimental platform sampling period is 0.02 seconds, and the iteration of algorithm is 20 times. It can implement the accuracy of the MPPT in real time. 
TABLE 2: Parameters of solar cell.

\begin{tabular}{|c|c|c|c|}
\hline $\begin{array}{l}\text { Maximum power } \\
\left(P_{\max }\right)\end{array}$ & $10 \mathrm{~W} \pm 3 \%$ & $\begin{array}{c}\text { Maximum system } \\
\text { voltage }\end{array}$ & $1000 \mathrm{VDC}$ \\
\hline Voltage at $P_{\max }$ & $17.5 \mathrm{~V}$ & Current at $P_{\max }$ & $0.57 \mathrm{~A}$ \\
\hline $\begin{array}{l}\text { Open-circuit } \\
\text { voltage }\left(V_{o c}\right)\end{array}$ & & $\begin{array}{l}\text { Short-circuit } \\
\text { current }\left(I_{\mathrm{sc}}\right)\end{array}$ & मோロロ \\
\hline
\end{tabular}

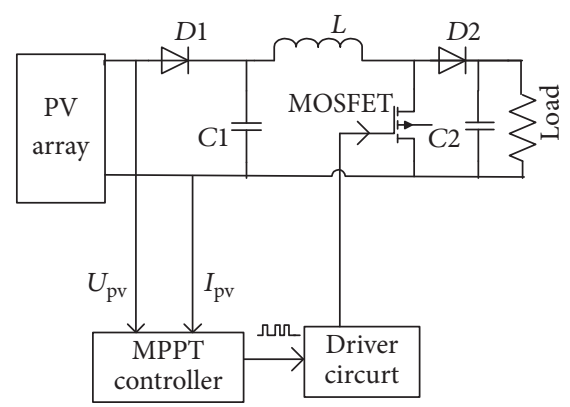

Figure 8: Experimental platform of PV MPPT.

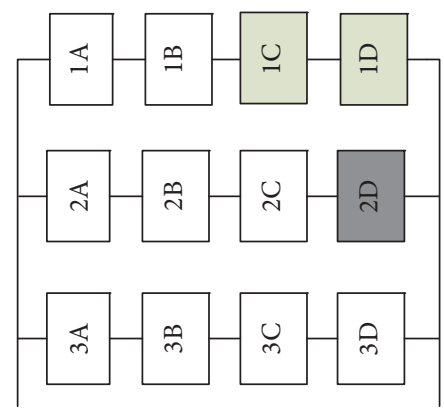

Figure 9: Configuration of PV array.

\subsubsection{Experiment Result of PSFLA Applied in MPPT for Photovoltaic Array}

(1) The Analysis and Comparison between D-P Characteristic Curve and V-P Characteristic Curve of PV. Using the MPPT experimental platform (Figure 7), the PV array is placed under uniform irradiance (in this case, $V_{\mathrm{OC}}$ and $I_{\mathrm{SC}}$ are $62 \mathrm{~V}$ and $1.27 \mathrm{~A}$, resp.), then a portion of the PV array is manually covered to simulate partial shading condition for MPPT experiment, and the initial sunshine is resumed after a period of time. A multiple peaks date in the partial shading condition was chosen to analyze as shown in Figure 10. It is obvious from Figure 10 that there are voltage repeat points in timevoltage curve. In this case, SIA cannot be used to optimize searching the power points based on $V-P$ characteristic curve. However, the time-duty cycle curve is linear change; there are no duty cycle repeat points. We compared the $V$ $P$ curve and $D$ - $P$ curve as shown in Figure 11, and these two curves have the same maximum power point. The maximum power was $31.2 \mathrm{~W}$ when duty cycle was $28.5 \%$ and voltage was $49.5 \mathrm{~V}$, respectively. So if we use SIA to optimize searching the power points based on $D-P$ characteristic curve, SIA can succeed in locating the global maximum power point.
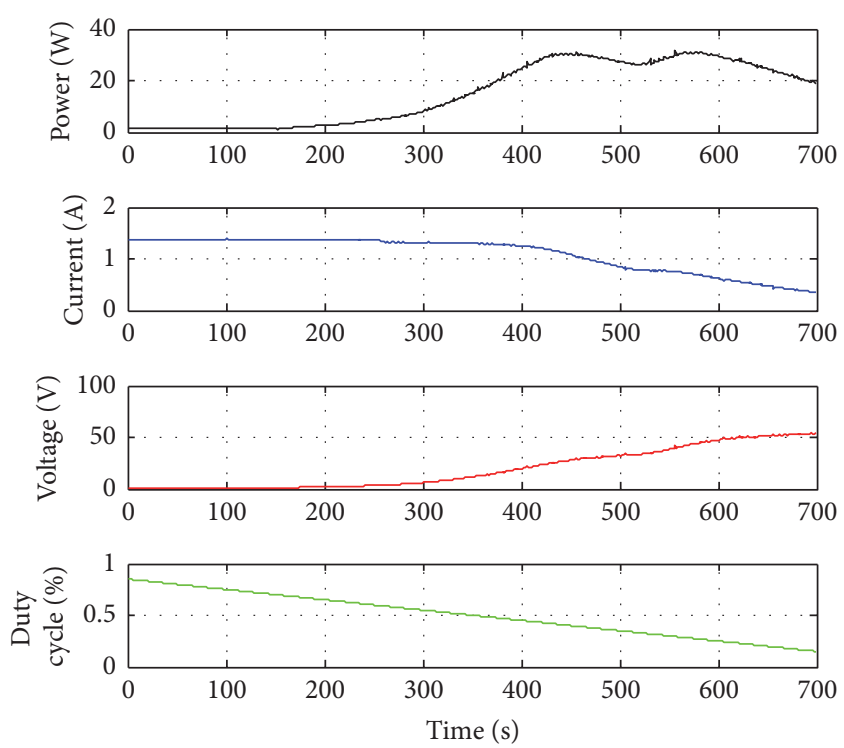

FIGURE 10: Real-time measurement data.

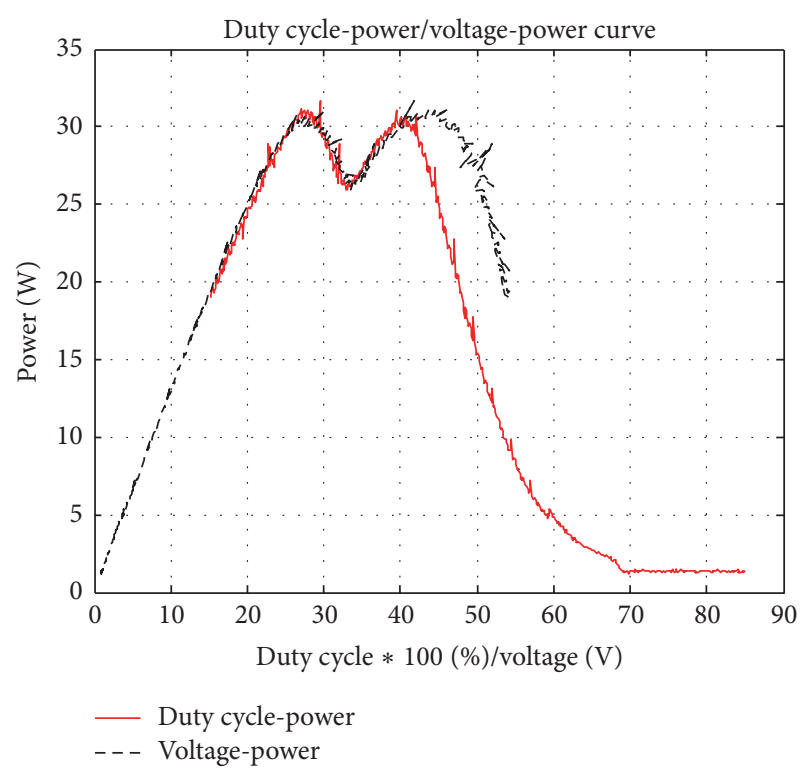

FIgUre 11: The comparison between $D-P$ and $V-P$ characteristic curve.

(2) The Experimental Results Based on the Static Multiple Peaks Curve of $P V$. After being preprocessed based on recursive least square filtering, the $D-P$ characteristic curve of Figure 11 is shown in Figure 12, where we can see that the measurement noise and outliers are suppressed effectively. Then, the PSFLA combined with recursive least square filtering will be adopted to locate the GP from multiple peaks under partial shading condition.

The real maximum power point was $31.2 \mathrm{~W}$ when duty cycle was $28.5 \%$ in Figure 12, but there was an outlier point (i.e., false maximum power point) $32.5 \mathrm{~W}$ when duty cycle was $29.8 \%$. If the conventional MPPT algorithm was adopted, it was easy to track and locate the false maximum 


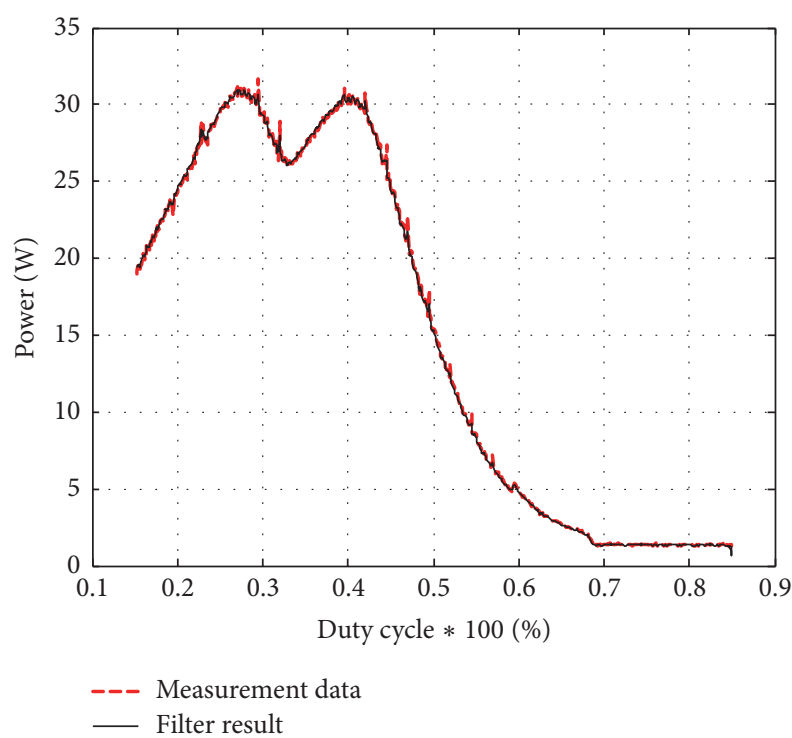

FIGURE 12: $D-P$ characteristics after least square of PV.

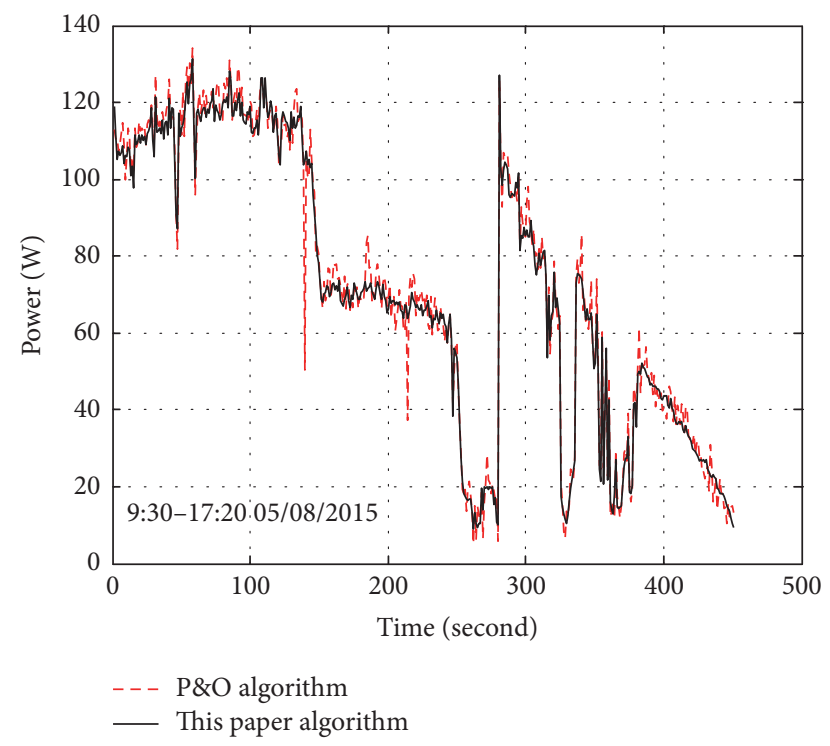

FIgURE 13: The experiment result of a full day.

power point instead of the real maximum power point. The experimental result indicated the PSFLA combined with recursive least square filtering correctly searched and located the real maximum power point.

(3) The Experimental Results of a Full Day. We used the proposed MPPT control strategy and the conventional P\&O (perturbation \& observation) method in MPPT dynamic experiment of a full day (i.e., 05/08/2015), respectively. The experimental and comparative results were shown in Figure 13. A total of 450 maximum power points were in Figure 13 with sunlight irradiation changes of 450 seconds (during 9:30-17:20 05/08/2015). The each dynamic maximum power point was located from static single or multiple peaks of $D$ - $P$ curves according to real irradiation changes.

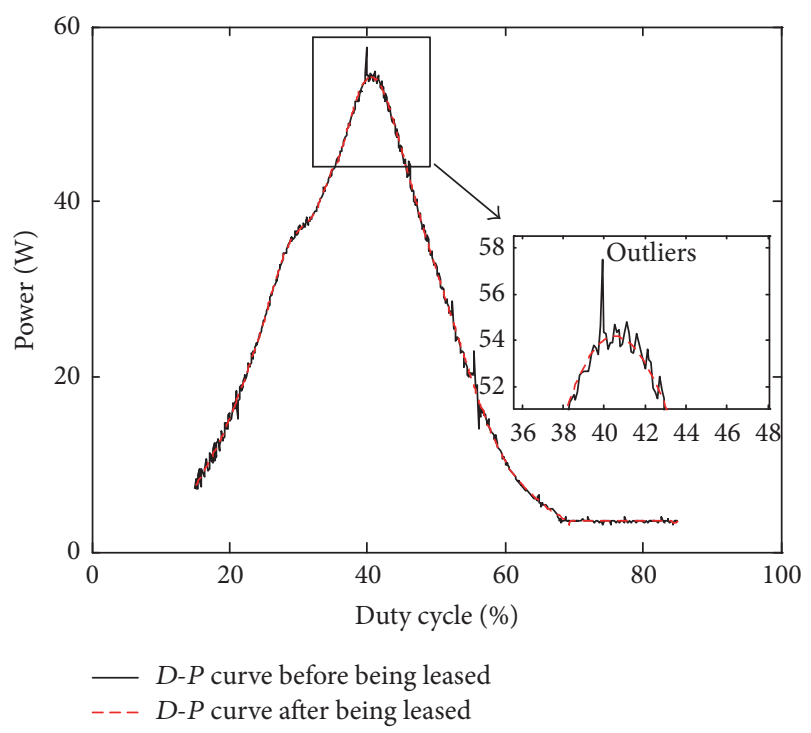

Figure 14: PV array $P$ - $D$ curves for test 1.

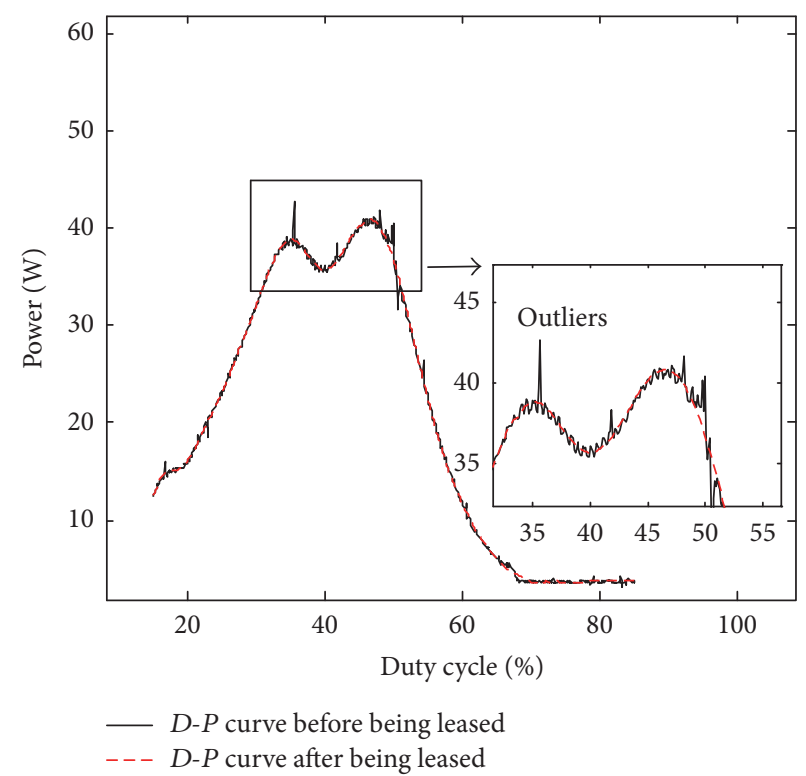

FIgURE 15: Photovoltaic array $P-D$ curves for test 2.

We can see that the conventional P\&O MPPT method has the problem of measurement noise and outliers which were solved by the proposed MPPT control strategy in this paper perfectly. There were larger measurement errors in the conventional P\&O MPPT method and failures in 140 seconds and 214 seconds, respectively, because of outliers. This indicates that the proposed MPPT control strategy in this paper can improve the accuracy of MPPT.

\subsubsection{Experiment and Comparison between PSLFA and PSO Algorithm}

(1) The Parameters Setting of Algorithms. In order to verify the advantage of PSLFA algorithm, we have experimented with 

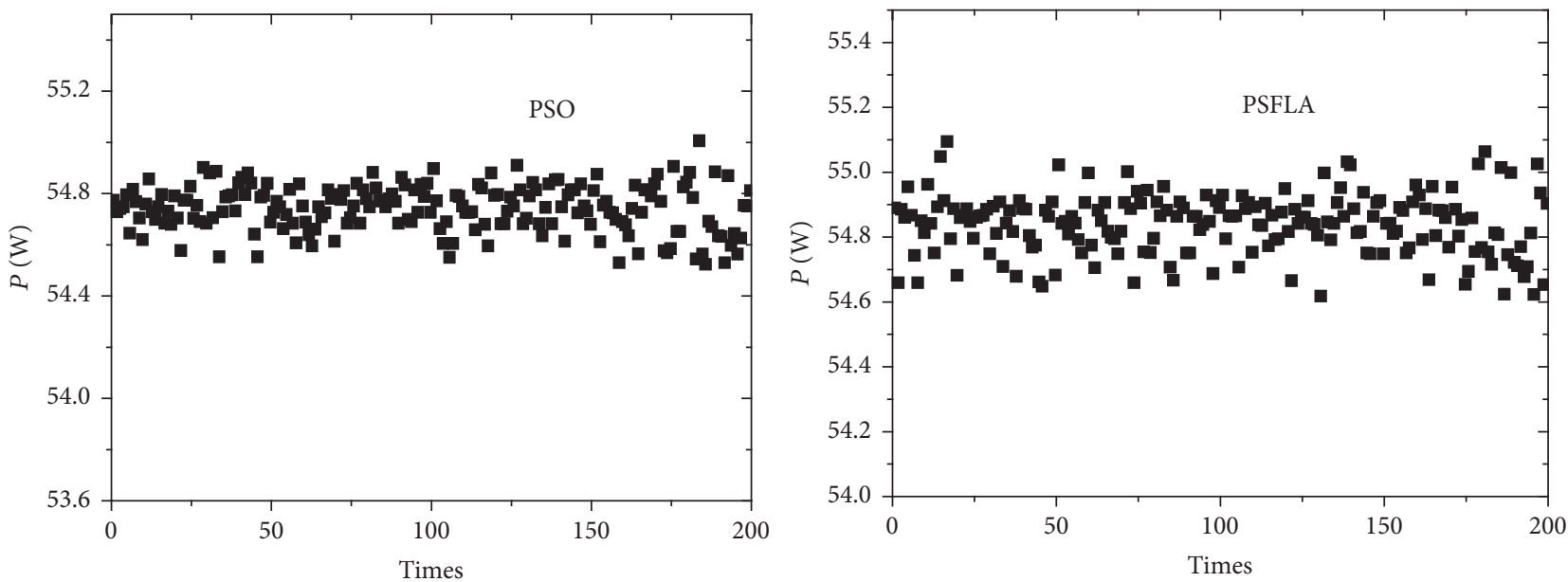

(a) Power tracking accuracy
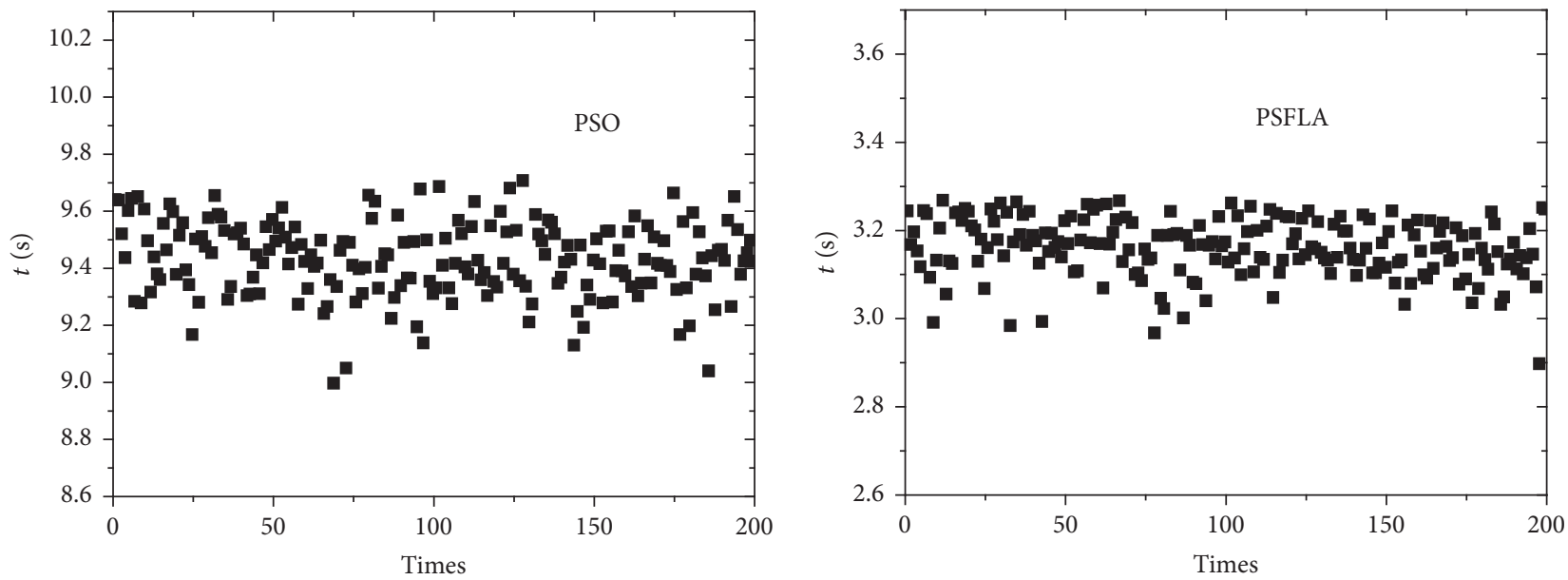

(b) Algorithm running time

FIgURE 16: Experimental results for test 1.

PSLFA and PSO algorithm. The parameters of algorithms are as follows:

(a) Parameters of PSO algorithm: the particle position changed according to

$$
w(k)=w_{\max }-\left(\frac{\left(w_{\max }-w_{\min }\right)}{G}\right) * k,
$$

where $w_{\max }=0.9$ and $w_{\min }=0.1$. Particle maximum speed is limited to 0.1 .

(b) Parameters of PSFLA algorithm were described in Section 3.2.

(c) Termination strategy: if the value of control parameter remains unchanged in the continuous 20 iterations, then stop iteration.

(2) Comparison Result and Analysis. Each algorithm runs 200 times independently and compares each tracking accuracy and speed. Two kinds of experiment conditions are set up.

(a) Test 1: the module 1A of Figure 9 is blocked; the rest PV modules are illuminated normally. The PV array power-duty cycle $(P-D)$ curves are shown in Figure 14. The maximum power point of $\mathrm{PV}$ array is about $54 \mathrm{~W}$.

(b) Test 2: the modules $1 \mathrm{~A}, 1 \mathrm{~B}$, and $2 \mathrm{~A}$ of Figure 9 are blocked; the rest PV modules are illuminated normally. Photovoltaic array $P-D$ curves are shown in Figure 15. The maximum power point of PV array is about $41 \mathrm{~W}$.

The power tracking accuracy and tracking time for two tests running 200 times are shown in Figures 16 and 17, respectively. Experiment summary results for two tests are shown in Tables 3 and 4, respectively.

It can be seen from Figure 16, Table 3, Figure 17, and Table 4 that PSFLA and PSO finally succeeded in overcoming the influence of multipower extremes and outliers, converging to the global maximum power point. Two algorithms have the same power tracking accuracy, but the running time of PSFLA algorithm is minimal. It shows that response speed of PSFLA algorithm is faster than PSO algorithm. Thus, the proposed MPPT control strategy based on PSFLA can suppress the measurement noise effectively and improve the PV array efficiency. 

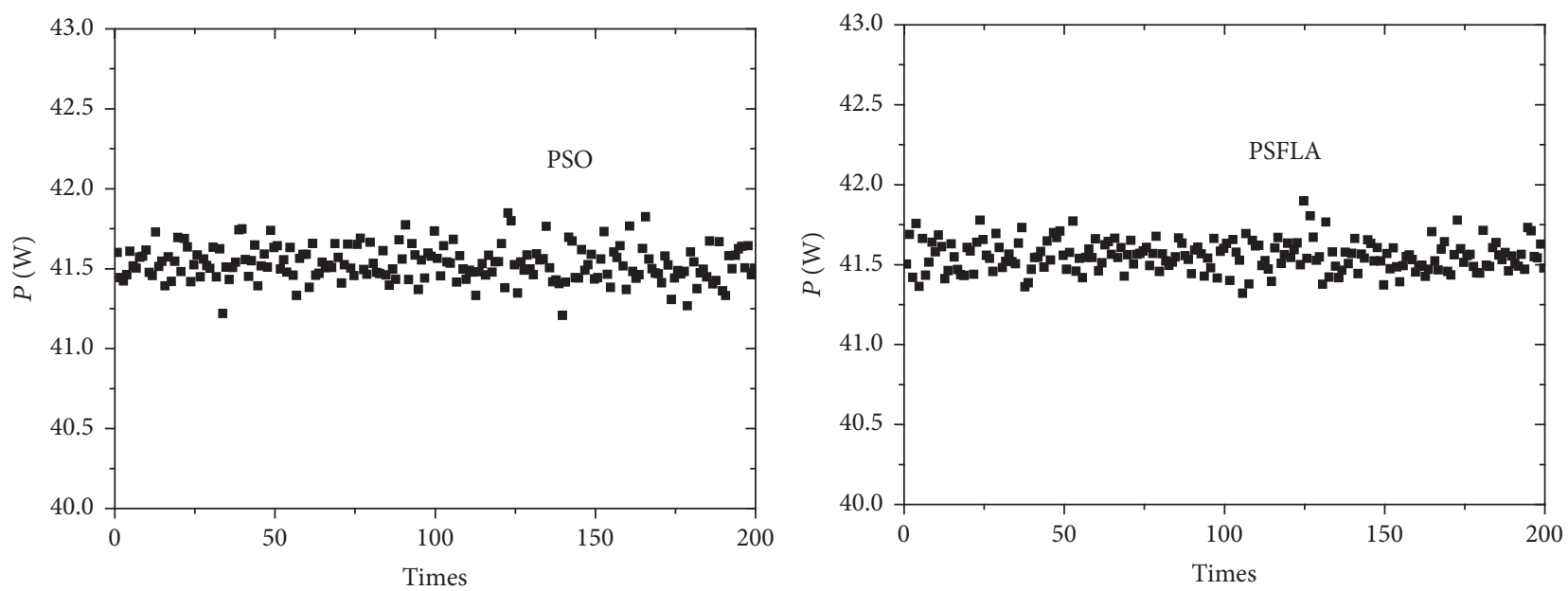

(a) Power tracking accuracy
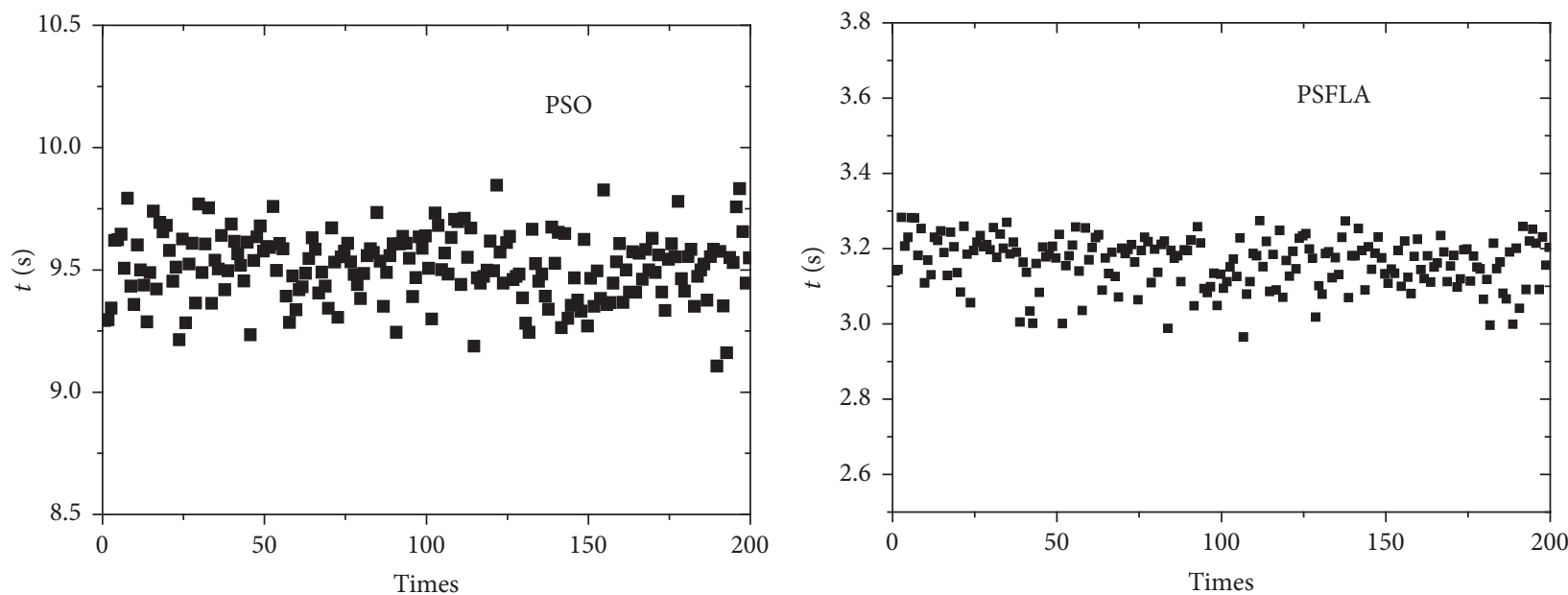

(b) Algorithm running time

FIGURE 17: Experimental results for test 2.

TABLE 3: Experimental summary results for test 1.

\begin{tabular}{|c|c|c|c|c|}
\hline \multirow{2}{*}{ Algorithms } & \multicolumn{3}{|c|}{ Power tracking accuracy $(\mathrm{W})$} & \multirow{2}{*}{$\begin{array}{c}\text { Average } \\
\text { running time (s) }\end{array}$} \\
\hline & Minimum & Maximum & Mean value & \\
\hline PSO & 54.5195 & 55.0025 & 54.7381 & \\
\hline PSFLA & 54.6151 & 55.0915 & 54.8358 & 3.1588 \\
\hline
\end{tabular}

TABLE 4: The experiment summary result for test 2.

\begin{tabular}{|c|c|c|c|c|}
\hline \multirow{2}{*}{ Algorithms } & \multicolumn{3}{|c|}{ Power tracking accuracy $(\mathrm{W})$} & \multirow{2}{*}{$\begin{array}{c}\text { Average } \\
\text { running time (s) }\end{array}$} \\
\hline & Minimum & Maximum & Mean value & \\
\hline PSO & 41.2029 & 41.8425 & 41.5238 & \\
\hline PSFLA & 41.3149 & 41.8933 & 41.5482 & 3.5605 \\
\hline
\end{tabular}

\section{Conclusions}

In this paper, a new kind of MPPT strategy based on the PSO improved shuffled frog leaping algorithm (PSFLA) was proposed in this paper. Through the simulation and the experiment, we can get the following:

(1) The proposed PSFLA algorithm has better performance in both the accuracy and speed than the PSO and SFLA algorithm.

(2) The MPPT control strategy based on PSFLA can locate the global peak from multiple peaks under partial shading condition effectively.

(3) As the problem of voltage repeat points, we take the duty cycle as the control variable. The MPPT strategy based on PSFLA combined with recursive least square filter can overcome the effect of the complex environments effectively, locate the global maximum power under the complex environments, and improve the search accuracy of MPPT.

\section{Conflicts of Interest}

The authors declare that they have no conflicts of interest. 


\section{Acknowledgments}

This work was supported by National Natural Science Foundation of China (no. 51467013).

\section{References}

[1] Z. Zhengming, C. Jian, and S. Xiaoying, Maximun Power Point Tracking Technology for Photovoltaic Power Generation, Publishing House of Electronics Industry, Beijing, China, 2012.

[2] H. Heydari-doostabad, R. Keypour, M. R. Khalghani, and M. H. Khooban, "A new approach in MPPT for photovoltaic array based on Extremum Seeking Control under uniform and nonuniform irradiances," Solar Energy, vol. 94, pp. 28-36, 2013.

[3] H. Heydari-doostabad, R. Keypour, M. R. Khalghani, and M. H. Khooban, "A new intelligent online fuzzy tuning approach for multi-area load frequency control: Self Adaptive Modified Bat Algorithm," International Journal of Electrical Power \& Energy Systems, vol. 31, no. 71, pp. 254-261, 2015.

[4] K. Ishaque and Z. Salam, "A review of maximum power point tracking techniques of PV system for uniform insolation and partial shading condition," Renewable \& Sustainable Energy Reviews, vol. 19, pp. 475-488, 2013.

[5] X. Nie and J. Lai, "A survey on tracking and control approaches for global maximum power point of photovoltaic arrays in partially shaded environment," Power System Technology, vol. 38, no. 12, pp. 3279-3285, 2014.

[6] A. M. Latham, R. Pilawa-Podgurski, K. M. Odame, and C. R. Sullivan, "Analysis and optimization of maximum power point tracking algorithms in the presence of noise," IEEE Transactions on Power Electronics, vol. 28, no. 7, pp. 3479-3494, 2013.

[7] B. O. Kang and J. H. Park, "Kalman filter MPPT method for a solar inverter," in Proceedings of the 2011 IEEE Power and Energy Conference at Illinois, PECI 2011, usa, February 2011.

[8] N. Xiaohua, "Research on the application of recursive least square estimate in photovoltaic system maximum power point tracking," Electrical Measurement \& Instrumentation, vol. 49, no. 563, pp. 49-52, 2012.

[9] M. Miyatake, M. Veerachary, F. Toriumi, N. Fujii, and H. Ko, "Maximum power point tracking of multiple photovoltaic arrays: a PSO approach," IEEE Transactions on Aerospace and Electronic Systems, vol. 47, no. 1, pp. 367-380, 2011.

[10] K. Ishaque and Z. Salam, "A deterministic particle swarm optimization maximum power point tracker for photovoltaic system under partial shading condition," IEEE Transactions on Industrial Electronics, vol. 60, no. 8, pp. 3195-3206, 2013.

[11] Y.-Y. Hong, A. A. Beltran, and A. C. Paglinawan, "A chaosenhanced particle swarm optimization with adaptive parameters and its application in maximum power point tracking," Mathematical Problems in Engineering, vol. 2016, Article ID 6519678, 19 pages, 2016.

[12] L. L. Jiang, D. L. Maskell, and J. C. Patra, "A novel ant colony optimization-based maximum power point tracking for photovoltaic systems under partially shaded conditions," Energy and Buildings, vol. 58, pp. 227-236, 2013.

[13] K. Sundareswaran, P. Sankar, P. S. R. Nayak, S. P. Simon, and S. Palani, "Enhanced energy output from a PV system under partial shaded conditions through artificial bee colony," IEEE Transactions on Sustainable Energy, vol. 6, no. 1, pp. 198-209, 2015.

[14] M. M. Eusuff and K. E. Lansey, "Optimization of water distribution network design using the shuffled frog leaping algorithm,"
Journal of Water Resources Planning and Management, vol. 129, no. 3, pp. 210-225, 2003.

[15] E. Elbeltagi, T. Hegazy, and D. Grierson, "Comparison among five evolutionary-based optimization algorithms," Advanced Engineering Informatics, vol. 19, no. 1, pp. 43-53, 2005.

[16] S. Jazebi, M. M. Hadji, and R. A. Naghizadeh, "Distribution network reconfiguration in the presence of harmonic loads: Optimization techniques and analysis," IEEE Transactions on Smart Grid, vol. 5, no. 4, pp. 1929-1937, 2014.

[17] A. Khorsandi, A. Alimardani, B. Vahidi, and S. H. Hosseinian, "Hybrid shuffled frog leaping algorithm and Nelder-Mead simplex search for optimal reactive power dispatch," IET Generation, Transmission \& Distribution, vol. 5, no. 2, pp. 249-256, 2011.

[18] S. Panda, A. Sarangi, and S. P. Panigrahi, "A new training strategy for neural network using shuffled frog-leaping algorithm and application to channel equalization," $A E U$ - International Journal of Electronics and Communications, vol. 68, no. 11, pp. 1031-1036, 2014.

[19] W. H. Cui, X. B. Liu, W. Wang, and J. Wang, "Survey on shuffled frog leaping algorithm," Control and Decision, vol. 27, no. 4, pp. 481-486, 493, 2012.

[20] J.-J. Li, B. Yu, and W.-P. Chen, "Improvement and simulation for shuffled frog leaping algorithm," Journal of System Simulation, vol. 26, no. 4, pp. 755-760, 2014.

[21] X. Li, J. P. Luo, M. R. Chen, and N. Wang, "An improved shuffled frog-leaping algorithm with extremal optimisation for continuous optimisation," Information Sciences, vol. 192, no. 1, pp. 143-151, 2012.

[22] Y. Shuying and Z. Hua, Swarm Intelligence and Bionic Calculation, Publishing House of Electronics Industry, Beijing, China, 2012. 


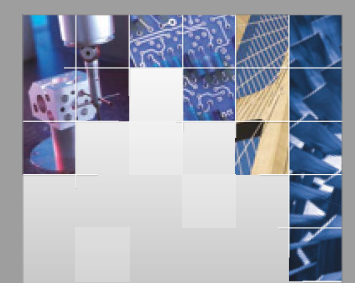

\section{Enfincering}
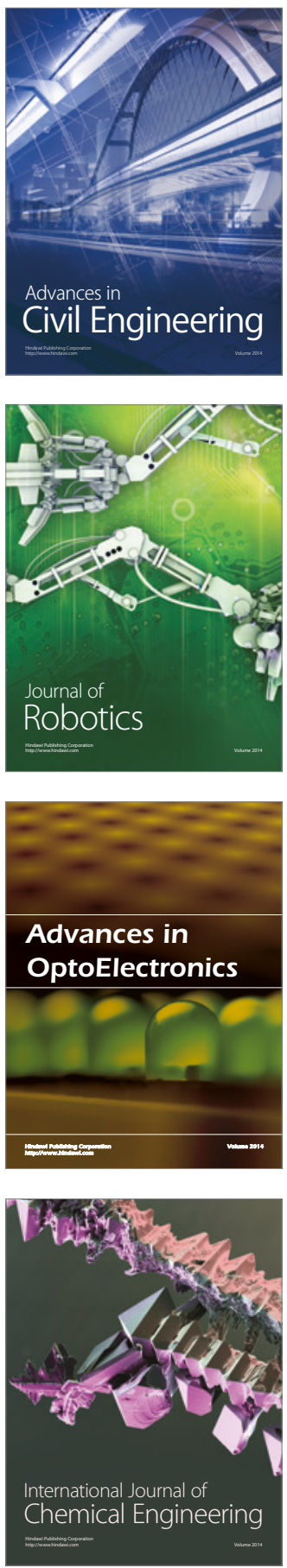

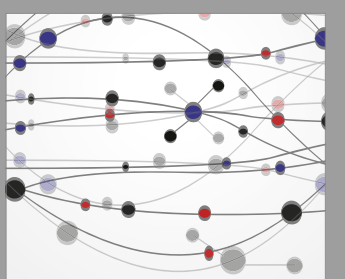

The Scientific World Journal

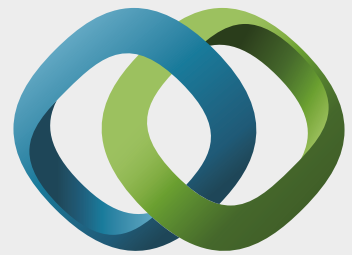

\section{Hindawi}

Submit your manuscripts at

https://www.hindawi.com
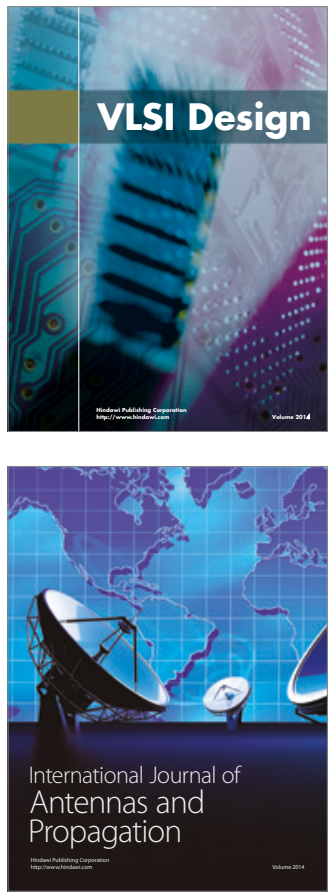

\section{Rotating}

Machinery
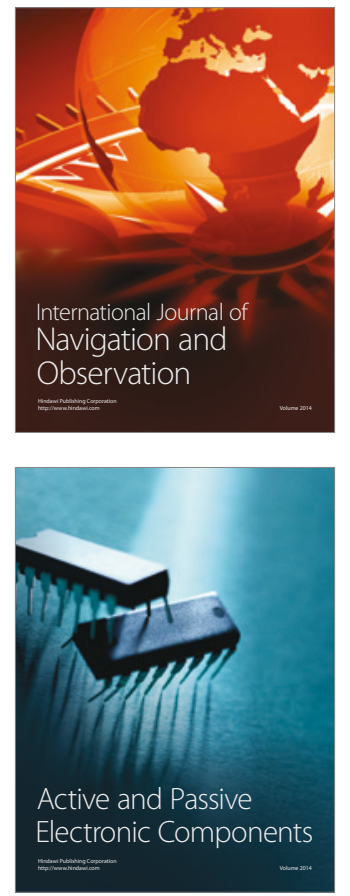
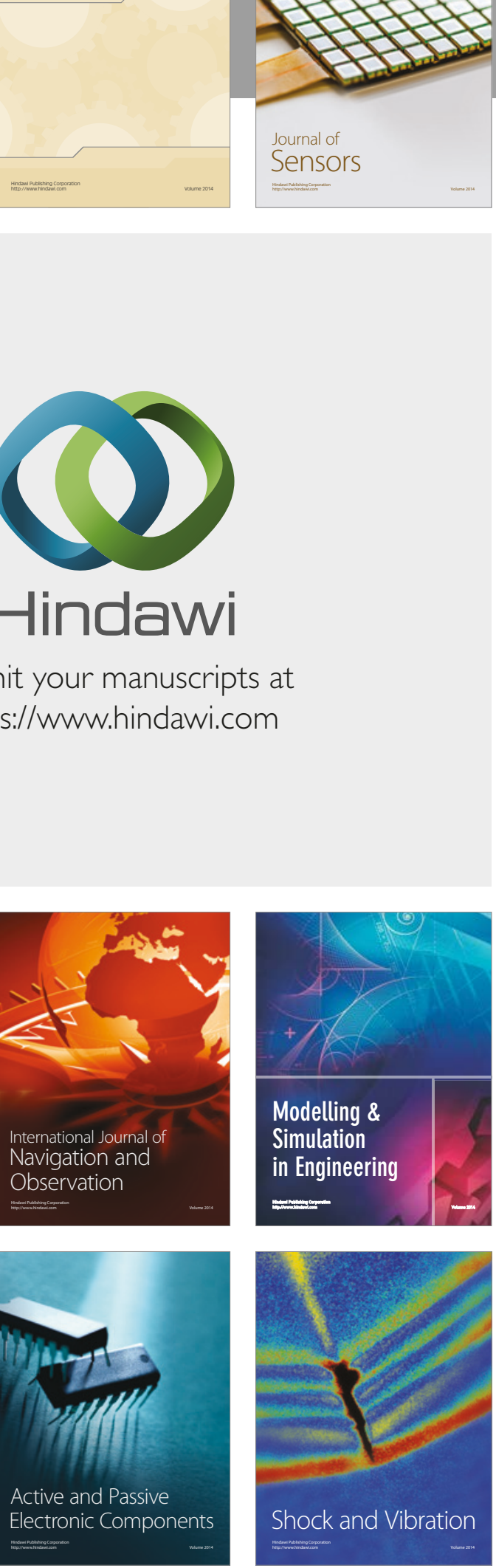
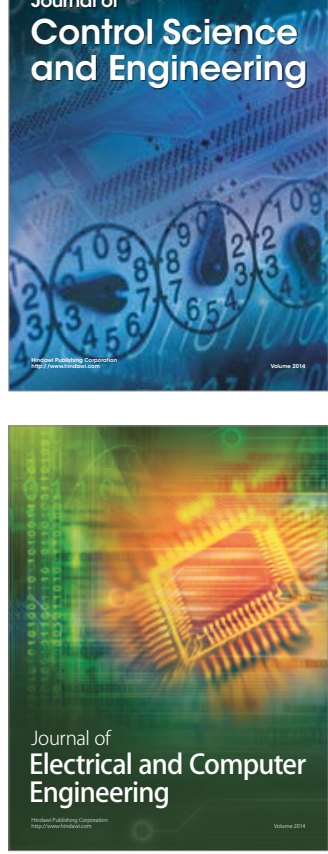

Distributed

Journal of

Control Science

and Engineering
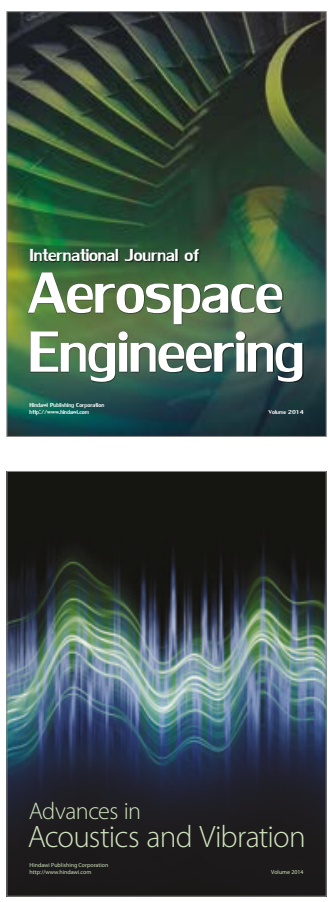

Sensor Networks 